\title{
FOCALISATION TEMPORELLE
}

\author{
S. BOURENNANE et B. FAURE \\ Laboratoire CEPHAG - ENSIEG - CNRS URA 346, Domaine Universitaire, BP. 46, \\ F-38402 St Martin d'Hères cedex, France
}

\begin{abstract}
Résumé : Pour caractériser des sources rayonnantes large bande à partir des signaux reçus sur un réseau de capteurs, plusieurs méthodes ont été développées à ce jour. Les solutions proposées sont essentiellement basées sur des moyennes temporelles (méthodes temporelles), spatiales et/ou fréquentielles (méthodes fréquentielles). Dans cette étude nous regroupons les méthodes fréquentielles en deux principales catégories (méthodes à sous-espace signal incohérent et méthodes à sous-espace signal cohérent). Ces diverses méthodes (temporelles et fréquentielles) de traitement d'antenne large bande sont appliquées à des signaux expérimentaux reçus sur une antenne en acoustique sous marine.
\end{abstract}

\begin{abstract}
Several methods have been proposed to caracterize the radiating wideband sources from the received signals on an antenna. The proposed methods are based in the temporal, spatial and/or frequential smoothing. In this paper, we regroup the frequential methods into two principal categories : the incoherent signal subspace methods and the coherent signal subspace methods. The different wideband array processing methods (temporal and frequential) are applied on the underwater experimental data.
\end{abstract}

\section{1- INTRODUCTION}

La localisation de sources rayonnantes bande large dans le domaine fréquentiel présente des difficultés. Ces difficultés se situent au niveau de la combinaison de toutes les informations contenues dans la bande d'analyse. En général, les méthodes développées utilisent soit le sous espace signal incohérent soit le sous espace signal cohérent. Pour la première méthode, le problème se pose au niveau de l'estimation du résultat final des directions des sources. De plus cette méthode n'arrive pas à éviter l'ambiguïté fréquence/angle due aux variations simultanées de la fréquence et de l'azimut. En effet, tous les couples "fréquence-angle" tel que $\mathrm{f}$. $\sin \theta=$ constante conduisent au même vecteur directionnel. Dans la deuxième méthode fréquentielle dite à sous espace signal cohérent, la combinaison d'informations se fait en amont de l'analyse spatiale en utilisant des matrices de transformation appelées opérateurs de focalisation. L'estimation de ces matrices nécessite des connaissances a priori sur les azimuts des sources, et par conséquent les performances de ces méthodes dépendent de cette initialisation ou connaissance.

Dans ce papier, nous nous intéressons à la localisation de sources large bande dans le domaine temporel. Cette méthode permet d'éviter les difficultés rencontrées avec les méthodes fréquentielles. Nous présentons les résultats obtenus sur des signaux expérimentaux d'acoustique sous marine en utilisant les méthodes fréquentielles et la méthode temporelle et nous dégageons des limites d'applications de ces méthodes.

\section{2 - POSITION DU PROBLEME}

On considère l'observation vectorielle $\underline{r}(t)=\left[r_{1}(t), \ldots, r_{N}(t)\right]^{T}$ mesurée sur les sorties des $N$ capteurs d'une antenne pendant une durée $\mathrm{T}$; où $\mathrm{r}_{\mathrm{i}}(\mathrm{t})$ représente le signal reçu sur le capteur $\mathrm{i}$ : 
$r_{i}(t)=\sum_{k=1}^{p} \alpha_{i_{k}} S_{k}\left(t-\tau_{i k}\right)+b_{i}(t) \quad-T / 2 \leq t \leq T / 2$

$S_{k}($.$) : le signal émis par la k^{\text {ième }}$ source ; $\tau_{i_{k}}$ : la durée de propagation source $k /$ capteur $i ; b_{i}(t): b r u i t$ additif sur le capteur $\mathrm{i} ; \alpha_{\mathrm{i}_{\mathrm{k}}}$ : amplitude complexe du signal émis par la source $\mathrm{k}$ et reçue sur le capteur $\mathrm{i}$.

\subsection{Méthodes fréquentielles}

En se plaçant dans les hypothèses classiques du traitement d'antenne [1] ; et en utilisant la notation matricielle; le vecteur des signaux reçus s'écrit dans le domaine fréquentiel (avec $\left.\alpha_{i_{k}}=\alpha_{i}=1\right)$.

$\underline{R}\left(f_{n}\right)=\underline{A}\left(f_{n}\right) \underline{S}\left(f_{n}\right)+\underline{B}\left(f_{n}\right)$ où $\underline{\underline{A}}\left(f_{n}\right)=\left[\underline{a} 1\left(f_{n}\right), \ldots, \underline{a}_{p}\left(f_{n}\right)\right]$ est la matrice de transfert entre les $P$ sources et les $\mathrm{N}$ capteurs de l'antenne à la fréquence $\mathrm{f}_{\mathrm{n}}$, avec :

$\underline{a_{k}}\left(f_{n}\right)=\frac{1}{\sqrt{N}}\left[1, e^{-j \varphi k n}, \ldots, e^{-j(N-1)} \varphi_{k n}\right]^{T}$ qui représente le vecteur directionnel de la source $k$.

La matrice interspectrale des signaux reçus à la fréquence $f_{n}$ (si les $B_{i}\left(f_{n}\right)(1 \leq i \leq N)$ sont non corrélés et de même variance $\left.\sigma^{2}\left(f_{n}\right)\right)$ est :

$\underline{\underline{\gamma}}\left(f_{n}\right)=\underline{\underline{A}}\left(f_{n}\right) \underline{\underline{\gamma}}_{S}\left(f_{n}\right) \underline{\underline{A}}^{+}\left(f_{n}\right)+\sigma^{2}\left(f_{n}\right) \underline{\underline{I}} \quad 1 \leq n \leq M$

\section{2-1-1 Traitements multi-bandes étroites}

Elle consiste à considérer la bande du signal comme une juxtaposition de bandes étroites considérées comme statistiquement indépendantes. En particulier, la localisation des sources large bande s'obtient en effectuant l'analyse utilisant les vecteurs propres de chaque matrice interspectrale, associée à chaque fréquence $\mathrm{f}_{\mathrm{n}}$ de la bande, puis sur chaque matrice on estime par la méthode du goniomètre les azimuts des sources par projection du modèle vecteur source construit à cette fréquence, sur le sous-espace signal ou sur son complément. On effectue ensuite la moyenne des différents résultats obtenus $Q_{A}(\theta)=\frac{1}{M} \sum_{n=1}^{M}\left[\left.\sum_{i=p+1}^{N} \underline{a}_{\theta}^{+}\left(f_{n}\right) \underline{V}_{i}\left(f_{n}\right)\right|^{2}\right]$

\section{2-1-2 Traitement utilisant le sous espace signal cohérent :}

L'estimation des azimuts des sources s'obtient par projection du modèle vecteur source construit à la fréquence centrale fo de la bande dite de focalisation, sur les grands vecteurs propres de la matrice interspectrale moyenne définie par:

$$
\hat{\psi}\left(f_{0}\right)=\frac{1}{M} \sum_{n=1}^{M} \underline{H}\left(f_{0}, f_{n}\right) \chi\left(f_{n}\right) \underline{H}^{+}\left(f_{0}, f_{n}\right)
$$

où $\underline{\underline{H}}\left(f_{0}, f_{n}\right)$ est l'opérateur de focalisation [1].

les directions des sources sont données par les minima de :

$Q(\theta)=\sum_{i=p+1}^{N}\left|\underline{a}_{0}^{+}\left(f_{0}\right) \widehat{V}_{i}\left(f_{0}\right)\right|^{2}$

\section{2-2 Méthode temporelle}

Les performances des méthodes fréquentielles présentées ci-dessus dépendent des paramètres des sources [1]. Pour améliorer la localisation des sources large bande des méthodes temporelles ont été développées. Ces méthodes utilisent la matrice de covariance spatio-temporelle des signaux reçus. Afin de remédier aux problèmes d'ambiguités dues aux variations simultanées de la fréquence et de l'angle, le vecteur spatio-temporel large bande [2] est utilisé :

$$
\underline{r}_{k}^{T}=\left[\underline{r}^{T}\left(t_{k}\right), \ldots \ldots, \underline{r}^{T}\left(t_{k}-(H-1) t_{0}\right)\right](N \times H)
$$


ave $\underline{r}^{\mathrm{T}}\left(\mathrm{t}_{\mathrm{k}}-(\mathrm{i}-1) \mathrm{t}_{0}\right)=\left[\mathrm{r}_{1}\left(\mathrm{t}_{\mathrm{k}}-(\mathrm{i}-1) \mathrm{t}_{0}\right), \ldots \ldots \ldots, \mathrm{r}_{\mathrm{N}}\left(\mathrm{t}_{\mathrm{k}}-(\mathrm{i}-1) \mathrm{t}_{0}\right)\right]$

où $\quad \mathrm{i}=1 \mathrm{à} H ; \mathrm{k}=1$ à $\mathrm{K}$ désigne le nombre de réalisations, $\mathrm{H}$ désigne le nombre de points d'échantillonnage considérés sur chaque sortie des capteurs de l'antenne.

Sous l'hypothèse de non corrélation entre les bruits et le signal utile, et les composantes du vecteur bruit spatio-temporel sont indépendantes et de même puissance $\sigma^{2}$ (bruit blanc spatialement et temporellement) la matrice de covariance $\Gamma_{\mathrm{k}}$ s'écrit : $\Gamma_{\mathrm{k}}=\Gamma_{\mathrm{s}}^{\mathrm{k}}+\sigma^{2} \mathrm{I}$

où I est une matrice identité (N.HxN.H). On définit le sous espace source et son complément par :

$$
\underline{\mathrm{V}}_{\mathrm{s}}=\left[\underline{\mathrm{v}}_{1}, \ldots \ldots, \underline{\mathrm{v}}_{\mathrm{p}}\right] \quad \text { avec } \mathrm{\rho}<\mathrm{P}(\mathrm{N}+\mathrm{H})
$$

$\underline{\mathrm{V}}_{\mathrm{B}}=\left[\underline{\mathrm{v}}_{\rho}+1, \ldots \ldots, \underline{\mathrm{v}}_{\mathrm{NH}}\right]$

Parmi les différents estimateurs possibles qui peuvent être utilisés pour obtenir les angles d'arrivée, nous retenons :

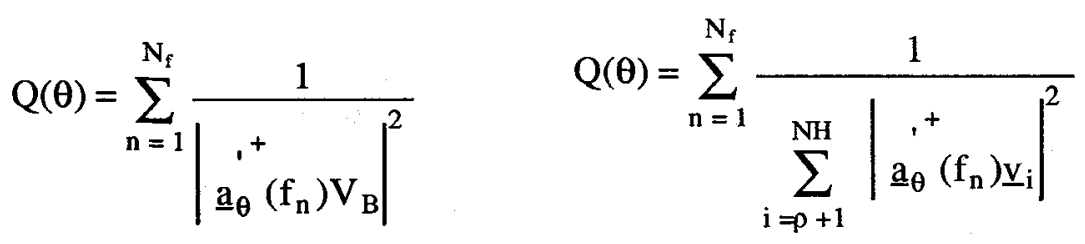

où Nf est le nombre de fréquences choisies dans la bande d'analyse;

$$
\underline{\mathrm{a}}_{\theta}(\mathrm{f})=\frac{1}{\sqrt{\mathrm{H}}}\left[1 \mathrm{e}^{-\mathrm{j} \varphi_{0}} \ldots . . \mathrm{e}^{-\mathrm{j}(\mathrm{H}-1) \varphi_{0}}\right]^{\mathrm{T}} \underline{\mathrm{a}}_{\theta}(\mathrm{f})
$$

avec $\varphi 0=2 \pi \mathrm{ft} 0$

\section{3 - APPLICATION A DES SIGNAUX EXPERIMENTAUX}

Nous avons pu mettre en oeuvre les méthodes fréquentielles et temporelle de localisation large bande. Pour cela nous avons utilisé les signaux provenant d'une campagne en mer [1] organisée par le GERDSM. La réception des signaux est faite sur une grande antenne horizontale. Nous considérons la bande de fréquence des signaux à traiter, définie par la bande du signal d'une source coopérative connue. Cette bande est de $20 \mathrm{~Hz}$ située autour de $82 \mathrm{~Hz}$.

A l'aide de la tranformée de Fourier rapide, la bande du signal est divisée en 100 fréquences, à chaque fréquence la matrice spectrale est estimée à partir des signaux reçus sur dix capteurs (1 à 10) de l'antenne. Ces matrices sont estimées en utilisant la méthode du périodogramme lissée avec un $\mathrm{B} \mathrm{e}=20$.

- Afin d'estimer le nombre de sources P, les critères AIC-MDL [1] ont été appliqués à la fréquence de focalisation $\mathrm{f}_{0}=82 \mathrm{~Hz}$. Les deux critères ont conduit au même résultat $\mathrm{P}=2$.

- Pour estimer les azimuts des sources détectées, nous avons utilisé les méthodes fréquentielles et temporelle. 
La figure 1 montre les différents résultats, sous forme de courbes de niveaux, obtenus pour l'analyse à toutes les fréquences. Nous constatons des fluctuations d'azimuts autour des valeurs moyennes : $3^{\circ}$ et $7^{\circ}$.

En utilisant la méthode de focalisation avec les directions initiales choisies $\theta_{1}=3^{\circ} \mathrm{et} \theta_{2}=7^{\circ}$. Comme précédemment, nous présentons (figure 2) les résultats obtenus.

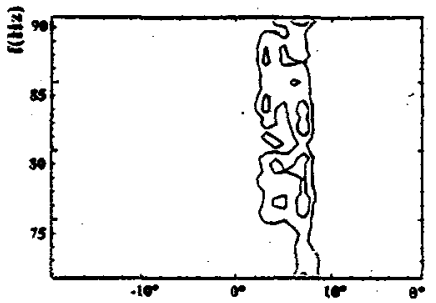

Figure 1 : Courbes de niveaux

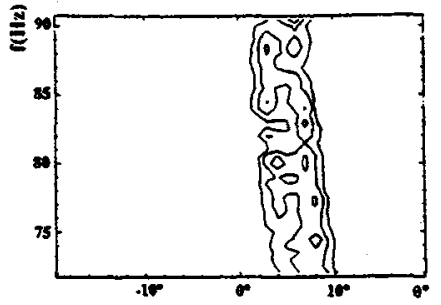

Figure 2 : Courbes de niveaux après focalisation.

Nous constatons, après focalisation que la dispersion des azimuts autour des valeurs moyennes est beaucoup plut petite, ce qui permet de conclure à une robustesse au bruit de la méthode de focalisation. Cette amélioration est due au moyennage cohérent effectué sur l'ensemble des fréquences de la bande.

La figure 3 montre les résultats obtenus par la méthode temporelle. La matrice de covariance est estimée à partir des mêmes données avec $\mathrm{N}=10$ et $\mathrm{H}=7$. Nous constatons que les résultats obtenus par cette méthode sont identiques à ceux obtenus par la méthode de focalisation fréquentiel

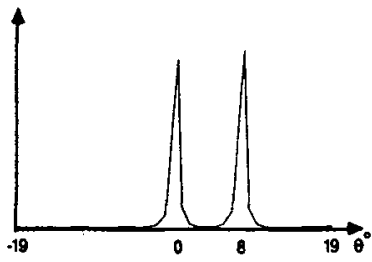

Figure 3 : Azimuts des sources obtenus par la méthode temporelle.

\section{CONCLUSION}

Cette étude, qui traite de la localisation de sources rayonnantes large bande, a permis de comparer les différentes méthodes fréquentielles et temporelle développées à ce jour.

Les résultats obtenus à partir des signaux expérimentaux montrent que la méthode de focalisation est plus robuste au bruit que la méthode multi-bandes étroites. La méthode temporelle conduit aux mêmes résultats que la méthode de focalisation (fréquentielle).

La méthode de focalisation nécessite des connaissances a priori des directions des sources et un temps de calcul important. La méthode temporelle est plus simple à utiliser dans le cas de sources non corrélées.

\section{BIBLIOGRAPHIE}

[ 1] S. BOURENNANE, Traitement d'antenne à large bande de fréquence. Thèse de Doctorat de I'INPG- CEPHAG 1990.

[ 2] K.M. BUCKLEY, L.J. GRIFFITHS, Broad band signal-subspace spatial-spectrum (BASS-ALE) estimation, IEEE ASSP, vol. 36, p. 953-964, July 1988. 